\title{
Global existence and exponential decay for hyperbolic dissipative relativistic fluid theories
}

Heinz-Otto Kreiss

Department of Mathematics, The University of California, Los Angeles, CA

90024, USA.

Gabriel B. Nagy, Omar E. Ortiz, and Oscar A. Reulaf

Fa.M.A.F. - U.N.C., Dr. Medina Allende y Haya de la Torre, Ciudad Universitaria, (5000) Córdoba, Argentina.

\begin{abstract}
We consider dissipative relativistic fluid theories on a fixed flat, globally hyperbolic, Lorentzian manifold $\left(\mathbf{R} \times \mathbf{T}^{3}, g_{a b}\right)$. We prove that for all initial data in a small enough neighborhood of the equilibrium states (in an appropriate Sobolev norm), the solutions evolve smoothly in time forever and decay exponentially to some, in general undetermined, equilibrium state. To prove this, three conditions are imposed on these theories. The first condition requires the system of equations to be symmetric hyperbolic, a fundamental requisite to have a well posed and physically consistent initial value formulation. The second condition is a generic consequence of the entropy law, and is imposed on the non principal part of the equations. The third condition is imposed on the principal part of the equations and it implies that the dissipation affects all the fields of the theory. With these requirements we prove that all the eigenvalues of the symbol associated to the system of equations of the fluid theory have strictly negative real parts, which in fact, is an alternative characterization for the theory to be totally dissipative. Once this result has been obtained, a straight forward application of a general stability theorem due to Kreiss, Ortiz, and Reula, implies the results above mentioned.
\end{abstract}

\footnotetext{
${ }^{1}$ Researcher of CONICET
} 


\section{INTRODUCTION}

In recent years there has been a substantial improvement on our understanding on how a proper description of dissipative fluids can be incorporated in the framework of the theory of relativity. Dissipative relativistic fluid theories satisfying an entropy law, and having a well posed (symmetric hyperbolic), and causal initial value formulation have been presented [1, 2, 3].

An important result on the physical meaning of all these hyperbolic theories was obtained recently [4, 氤]. It was shown that certain constitutive relations between the variables in the hyperbolic fluid theories, which have a clear physical meaning, approach in their time evolution the values predicted by the simplest covariant generalizations of the Navier Stokes fields. This result is based in a fundamental hypothesis namely, that the solution of the hyperbolic fluid field equations exists and remains smooth and small during a long enough time interval, such that the relaxation to near Navier-Stokes behavior occurs. This is a very important check for these fluid theories, since at microscopic scales they are substantially different from the usual Navier-Stokes theories in the following sense: for the last ones, one expects to have smooth global solutions for all smooth initial data sets, as has been proved in lower dimensions [6], while for the former ones, one expects the developing of discontinuities in the form of shock waves for crispy enough initial data. Thus, we can only hope to find a neighborhood of equilibrium data for which global solutions exist and so, where the departures from Navier-Stokes are uniformly small.

The purpose of the present work is to look for conditions under which the fundamental hypothesis mentioned above is satisfied. To this end, we apply a theorem [7] which is a generalization to the case of partial differential equations of the Ljapunov stability theorem for ordinary differential equations. This generalization holds for hyperbolic systems such that the eigenvalues of their associated symbols have all strictly negative real parts which, as we shall see, is the case for the hyperbolic dissipative fluids.

In order to apply this general stability theorem, three conditions are imposed on the dissipative hyperbolic fluid theories. The first condition requires the symmetric hyperbolicity of the system of equations; a fundamental requisite to have a well posed and physically consistent initial value formulation. As we shall see, the symmetry is an automatic property of these theories, but the hyperbolicity has to be required. We also include in this condition that the spacetime manifold is $\mathbf{R} \times \mathbf{T}^{3}$ with a flat metric (which is the more restrictive assumption). The other two conditions are of a generic type in the sense that all fluid systems, except for very specific and isolated ones, satisfy them. Specifically, the second condition requires that the nonprincipal part of the system of equations, which is responsible for the dissipation, satisfy certain negative-semidefiniteness condition. This condition assures that all perturbations to an equilibrium state, which are not tangent to the equilibrium 
submanifold, do dissipate towards equilibrium, and this is manifested by the fact that they make a positive definite contribution to the entropy. The third condition is a requirement on the principal part of the system of equations, and it means that the presence of dissipation affects all the fields of the theory, in the sense of not allowing for a decoupled set of fields with its own evolution not being driven by dissipation. Both, the second and the third conditions, have already been required in the literature with the aim of characterizing the equilibrium states.

These conditions allow us to apply the theorem proved in [7], which implies not only the global existence of solutions, but also their exponential decay to equilibrium for initial data near enough, in some appropriate norm, to equilibrium data.

The plan of the paper is as follows: In Sec. II we briefly introduce the fundamental aspects of the fluid theories, and state in detail the character of the conditions we impose on them. In Sec. III we state and prove our main result. Finally, in Sec. IV we present the conclusions.

\section{DISSIPATIVE RELATIVISTIC FLUID THEORIES}

In this section, following [3], we introduce the dissipative relativistic fluid theories. After introducing them, we describe the properties of these fluids needed to prove stability.

We assume that a fluid state is characterized by a finite collection of space-time tensor fields. Let $\varphi^{A}$ denote these fields, where upper case indices stand for the entire set of tensor indices represented in this collection of fields. So we refer to $\varphi^{A}$ as a point in the space of fluid states $\mathcal{S}$. Lower case indices will denote spacetime indices. Repeated indices indicate contraction as usual in the abstract index notation. We restrict consideration to the fluid theories in which the field equations take the form

$$
M_{A B}^{a} \nabla_{a} \varphi^{B}=-I_{A B} \varphi^{B} .
$$

where $M_{A B}^{a}$ and $I_{A B}$ are smooth functions of the fluid fields $\varphi^{A}$ and the space-time metric.

We say that system (11) is symmetric hyperbolic if $M_{A B}^{a}=M_{(A B)}^{a}$ and there exists a timelike-future directed $u^{a}$ such that $N_{A B} \equiv-u_{a} M_{A B}^{a}$ is positive definite. This is a sufficient condition to have a well posed initial value formulation.

On physical grounds, to have as maximum speed of propagation the speed of light, the stronger condition of causality, that is, $-u_{a} M_{A B}^{a}$ is positive definite for all future-directed timelike vector $u^{a}$, is usually required. This means that the characteristic surfaces of system (1) are inside the null cone given by the spacetime metric. To prove global existence and decay only the weaker condition of hyperbolicity is needed.

We analize now the structure of the equilibrium states. Following [2, 3] we say that a fluid state $\varphi^{A}$, solution of the dynamical system of equations, is a strict equi- 
librium state if its time reverse is also a solution. We denote a strict equilibrium state by $\varphi_{0}^{A}$, and assign a subindex zero to any tensor evaluated at an strict equilibrium state. This definition implies that $I_{0 A B} \varphi_{0}^{B}=0$. More generally, we say the $\varphi^{A}$ is a momentary equilibrium state if $I_{A B} \varphi^{B}=0$. These states are called equilibrium states because their entropy production vanishes, and momentary because if this condition holds at a certain time, it will not necessarily hold in subsequent times

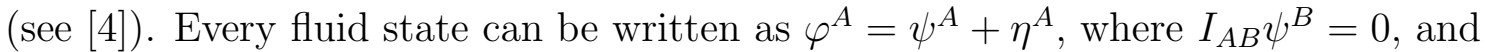
$\eta^{A}$ is such that $I_{A B} \eta^{B}=0$ implies $\eta^{A}=0$. We call $\psi^{A}$ the momentary equilibrium part of the fluid state.

Below we state the three conditions imposed on these fluid theories that will be used to show global existence and exponential decay.

1. The fluid system of equations (1) is symmetric hyperbolic and the space-time is $\left(\mathbf{R} \times \mathbf{T}^{3}, g_{a b}\right)$, where $\mathbf{T}^{3}$ denotes a three dimensional torus and $g_{a b}$ is a flat metric.

2. The tensor $I_{0 A B}$ must be symmetric and positive semidefinite.

3. The map $\mathcal{F}_{K}: \mathcal{S}_{\psi} \rightarrow \mathcal{S}^{*}$ defined by $\mathcal{F}_{K}\left(\psi^{A}\right) \equiv K_{a} M_{0 A B}^{a} \psi^{B}$, is injective for all space-time vectors $K^{a} \neq 0$; where $\mathcal{S}^{*}$ denotes the dual of the space of fluid states and $\mathcal{S}_{\psi}$ the subspace of momentary equilibrium states.

The first condition is the more restrictive and more work has to be done in order to weaken it. It would be interesting to treat physically relevant boundary conditions and arrive to similar results. The other two conditions are not very restrictive and all fluid systems, except for very specific and isolated ones, satisfy them.

The second requirement ensures that the effect of $I_{A B}$ in equation (11) is to dissipate, in the sense of tending to move non equilibrium states towards equilibrium as time grows. This is just a little stronger than the entropy condition in these fluid theories, that requires the entropy source to be non negative. This stronger condition was already considered in [2].

The third requirement is on the principal part of the equations. It implies that dissipation affects all the fields of the theory, in the sense of not allowing the existence of a decoupled subset of fields with its own evolution not being affected by dissipation. This requirement turns out to be equivalent (see appendix A), at least for the case of divergence type fluid theories, to one assumed in the literature [2] to show that the only possible strict equilibrium states are the constant states [8].

\section{GLOBAL EXISTENCE AND EXPONENTIAL DECAY}

In the theorem below we present our main result about global existence in time and decay to strict equilibrium. 
Theorem 1 Consider the Cauchy problem for system (1), corresponding to a hyperbolic divergence type fluid theory satisfying conditions 1-3. If the initial data is smooth and close enough in a $H^{p}\left(\mathbf{T}^{3}\right)$ Sobolev norm $(p>5)$ to the data corresponding to some strict equilibrium solution, then the solution is smooth, exists globally in time and decays exponentially to some strict equilibrium solution in the $H^{p}$ norm.

Proof: We define $N_{A B}=-u_{a} M_{A B}^{a}, N_{A B}^{a}=-q_{b}^{a} M_{A B}^{b}$ where $q_{a b}=g_{a b}+u_{a} u_{b}$ is the 3-metric in each hypersurface orthogonal to $u^{a}$ (assuming $u^{a} u_{a}=-1$ ). Assume that the initial data is close to some strict equilibrium state $\varphi_{0}^{A}$, then the fluid state is $\varphi^{A}=\varphi_{0}^{A}+\delta \varphi^{A}$. For the variable $\delta \varphi^{A}$, the system (11) becomes

$$
N_{A B} u^{a} \nabla_{a} \delta \varphi^{B}=N_{A B}^{a} \nabla_{a} \delta \varphi^{B}-I_{A B} \delta \varphi^{B} .
$$

Here the tensors $N_{A B}, N_{A B}^{a}$, and $I_{A B}$ are thought as functions of $\delta \varphi^{A}$. The condition (1) of Sec. II on the fluid theories implies that we can choose Cartesian coordinates $\left\{t, x^{j}\right\}$ on the space-time manifold $\mathbf{R} \times \mathbf{T}^{3}$ and a constant $u^{a}$ such that $\partial / \partial t=u^{a} \nabla_{a}$. Then the system (2) becomes

$$
N_{A B} \frac{\partial}{\partial t} \delta \varphi^{B}=N_{A B}^{j} \frac{\partial \delta \varphi^{B}}{\partial x^{j}}-I_{A B} \delta \varphi^{B} .
$$

In order to study solutions near $\delta \varphi^{A}=0$, it is convenient to introduce a parameter $\varepsilon$ to control the smallness of initial data. Thus, $\delta \varphi^{A}(t=0)=\varepsilon f^{A}\left(x^{j}\right)$ and the solution shall be written as $\delta \varphi^{A}=\varepsilon v^{A}$. As the tensors $N_{A B}^{j}$ and $I_{A B}$ are smooth functions of $\varepsilon v^{A}$, they can be written as

$$
\begin{aligned}
& N_{A B}^{j}=N_{0 A B}^{j}+\varepsilon N_{1 A B}^{j}, \\
& I_{A B}=I_{0 A B}+\varepsilon I_{1 A B} .
\end{aligned}
$$

With this decomposition the Cauchy problem for (3) is

$$
\begin{aligned}
& N_{A B} \frac{\partial v^{B}}{\partial t}=\left(N_{0 A B}^{j}+\varepsilon N_{1 A B}^{j}\right) \frac{\partial v^{B}}{\partial x^{j}}+\left(I_{0 A B}+\varepsilon I_{1 A B}\right) v^{B} . \\
& v^{A}(t=0)=f^{A}\left(x^{j}\right) .
\end{aligned}
$$

As there are periodic boundary conditions on the space coordinates $\left\{x^{j}\right\}, v^{A}$ can be expanded in Fourier series,

$$
v^{A}=\sum_{k^{j} \in \Omega} \hat{v}^{A}\left(k^{j}, t\right) e^{i \vec{x} \cdot \vec{k}},
$$

where $\Omega$ is the discrete set of Fourier frequencies.

We want to apply the stability theorem proved in [7] that, for completeness, we state in the appendix B. To prove the theorem 11, we consider the eigenvalues problem

$$
\lambda N_{0 A B} \widehat{\varphi}^{B}=\left(i k_{j} N_{0 A B}^{j}-I_{0 A B}\right) \widehat{\varphi}^{B} .
$$


Then, as explained in the appendix B, we only need to verify the following conditions.

(i) There is a constant $\delta>0$ such that the eigenvalues $\lambda\left(k^{j}\right)$ satisfy $\operatorname{Re}\{\lambda\} \leq-\delta$ for all $k^{j} \in \Omega, k^{j} \neq 0$.

(ii) For $k^{j}=0, \lambda(0) \leq-\delta$ or $\lambda(0)=0$.

(iii) As linear maps, the null space of $I_{1 A B}$ contains the null space of $I_{0 A B}$.

Conditions (ii) and (iii) are satisfied for these fluid theories, since the kernel of $I_{A B}$ is of constant dimension (see [2, 3]), and because condition (2) of Sec. II holds.

To prove (i), let $k^{j}$ be different from zero, then $k=\sqrt{k^{j} k_{j}} \geq$ const. $>0$. The eigenvalue problem (5) can be written as

$$
\left(-\frac{\lambda}{k} N_{0 A B}+i \frac{k_{j}}{k} N_{0 A B}^{j}\right) \widehat{\psi}^{B}=\left(\frac{\lambda}{k} N_{0 A B}+\frac{1}{k} I_{0 A B}-i \frac{k_{j}}{k} N_{0 A B}^{j}\right) \widehat{\eta}^{B} .
$$

Defining $K_{a}=-(\lambda / k) u_{a}+i\left(k_{j} / k\right) q_{a}^{j}$, this can be written as

$$
K_{a} M_{0 A B}^{a} \widehat{\psi}^{B}=\left(\frac{\lambda}{k} N_{0 A B}+\frac{1}{k} I_{0 A B}-i \frac{k_{j}}{k} N_{0 A B}^{j}\right) \widehat{\eta}^{B} .
$$

Since $K_{a} M_{0 A B}^{a}$ is injective, by condition (3), and a smooth function of $k_{j} / k$, there is a constant $c>0$ such that

$$
c N_{0 B C} \overline{\widehat{\psi}}^{B} \widehat{\psi}^{C} \leq K_{a} M_{0}^{a A}{ }_{B} K_{b} M_{0 A C}^{b} \overline{\widehat{\psi}}^{B} \widehat{\psi}^{C} .
$$

Then, contracting (6) with itself

$$
\begin{aligned}
& N_{0 B C} \overline{\widehat{\psi}}^{B} \widehat{\psi}^{C} \leq \\
& \frac{1}{c}\left(\frac{\bar{\lambda}}{k} N_{0}{ }^{A}{ }_{B}+\frac{1}{k} I_{0}{ }^{A}{ }_{B}+i \frac{k_{j}}{k} N_{0}^{j A}{ }_{B}\right)\left(\frac{\lambda}{k} N_{0 A C}+\frac{1}{k} I_{0 A C}-i \frac{k_{j}}{k} N_{0 A C}^{j}\right) \overline{\hat{\eta}}^{B} \widehat{\eta}^{C} .
\end{aligned}
$$

Perturbation theory of linear operators tells us that $\lambda\left(k^{j}\right) / k$ is uniformly bounded (see [9]), then we get for some positive $c^{\prime}$

$$
N_{0 B C} \overline{\widehat{\psi}}^{B} \widehat{\psi}^{C} \leq c^{\prime} N_{0 B C} \overline{\bar{\eta}}^{B} \widehat{\eta}^{C} .
$$

This inequality, together with the positive definiteness of $N_{0 A B}$ implies,

$$
N_{0 A B} \overline{\widehat{\varphi}}^{A} \widehat{\varphi}^{B} \leq \tilde{c} N_{0 A B} \overline{\hat{\eta}}^{A} \widehat{\eta}^{B}, \quad \tilde{c}>0 .
$$

Now, contracting (5) with $\bar{\varphi}^{A}$ and taking real part

$$
\begin{aligned}
\operatorname{Re}\{\lambda\} N_{0 A B} \overline{\bar{\varphi}}^{A} \widehat{\varphi}^{B} & =-I_{0 A B} \overline{\widehat{\varphi}}^{A} \hat{\varphi}^{B}=-I_{0 A B} \overline{\widehat{\eta}}^{A} \widehat{\eta}^{B} \\
& \leq-\tilde{\delta} N_{0 A B} \overline{\bar{\eta}}^{A} \widehat{\eta}^{B} \\
& \leq-\frac{\tilde{\delta}}{\tilde{c}} N_{0 A B} \bar{\varphi}^{A} \widehat{\varphi}^{B} .
\end{aligned}
$$


Here, $\tilde{\delta}>0$ exists because of the negative definiteness of $I_{0 A B}$ in the direction of $\widehat{\eta}^{A}$ (by condition (2)), and we have used (7) in the last line. We have thus shown that

$$
\operatorname{Re}\left\{\lambda\left(k^{j}\right)\right\} \leq-\delta<0, \quad \text { with } \delta=\frac{\tilde{\delta}}{\tilde{c}}>0 \text { and } k^{j} \neq 0 .
$$

\section{CONCLUSIONS}

In this work we have proved global existence and exponential decay to strict equilibrium of solutions, corresponding to initial data in a small enough neighborhood of strict equilibrium, for a generic dissipative relativistic fluid theory.

This result, in particular, verifies a fundamental hypothesis of previous works [回, 可 namely, the existence of solutions during a long enough time interval. The closeness of initial data to strict equilibrium is a natural limitation in the sense that, for large data, shock waves develop, which are a widely observed phenomena in nature. This occurs because these fluid systems are genuinely non-linear.

There are three questions related to the techniques used in this work and the possibility of improving on them. One is whether it is possible to extend the present result, or rather the general theorem used, to the case of non-constant equilibrium states, this is of vital importance if we want to consider non-flat backgrounds or even self-gravitating fluids. We think this is probable the case if we further assume that the theory has, at equilibrium, a conserved energy-that is a positive definite bilinear form in the tangent space of equilibrium states-as is usually the case for theories coming from a Hamiltonian formalism. The second possible extension is towards allowing non-compact Cauchy slices. There is another technique, introduced by Matsumura [10], which allows to study global existence and stability for some particular cases that ranges from hyperbolic heat conduction to relativistic superfluids [11]. This technique allows to treat the case of non-compact Cauchy slices, but can not be applied to the general systems considered in this work. Thus, it would be important to extend the theorem given in the appendix B to the case of non-compact Cauchy slices. The third extension is in the direction of boundary values problems. It is clear that one would like to use this theory to describe situations where the fluid is in a finite region of space, in that case the equations cease to be hyperbolic outside the region occupied by the fluid, and so a boundary value formulation is needed. Since Navier-Stokes fluids behave in a much more amenable way with regards to boundary conditions than perfect fluids, one would expect that these dissipative fluids will have that property too, making this an interesting, and perhaps tractable, problem. 


\section{ACKNOWLEDGMENT}

G.B.N., O.E.O. and O.A.R. thank ICTP for hospitality during part of the time of development of this work.

This work has been supported by grants from CONICOR, CONICET and SeCyT-UNC.

\section{APPENDIX A: EQUIVALENCE OF ASSUMPTIONS}

In this Appendix we prove the equivalence between the requirement (3) of Section 2 and a condition imposed in literature [2, 3]. We do this, for simplicity, in the case of divergence type fluid theories, and we assume that the reader is familiar with [2]. The condition under consideration allows to characterize the strict equilibrium states in such a way that they are precisely the same set of equilibrium states found for the standard Eckart theory.

The requirement 3 of Section 2 is the following: The map $\mathcal{F}_{K}: \mathcal{S}_{\psi} \rightarrow \mathcal{S}^{*}$ defined by $\mathcal{F}_{K}\left(\psi^{A}\right) \equiv K_{a} M_{0 A B}^{a} \psi^{B}$, is injective for all space-time vectors $K^{a} \neq 0$, where $\mathcal{S}^{*}$ denotes the dual of the space of fluid states and $\mathcal{S}_{\psi}$ the subspace of momentary equilibrium fluid states.

The equivalence between this condition and the one assumed in [2] follows from the following argument. The map $\mathcal{F}_{K}$ is injective so, $K_{a} M_{0 A B}^{a} \psi^{B}=0 \Rightarrow \psi^{A}=0$ where $\psi^{A}=\left(\psi, \psi^{a}, 0\right)$. Due to the definition of indices $A$ and $B$, the system of equations above represents a scalar equation, a vector equation, and a symmetric two indices tensor equation.

First, consider the scalar equation, the contraction of the vector equation with $\zeta^{a}$, and the contraction of the two indices tensor equation with $\zeta^{a} \zeta^{b}$. All this constitute a linear algebraic system of three scalar equations for variables $K_{a} \psi^{a}, K_{a} \zeta^{a} \psi$, and $2 K_{a} \zeta^{a} \zeta^{b} \psi_{b}$. The injectivity implies that the only solution for these three variables is zero and so, the determinant of the coefficient matrix is different from zero. Conversely, if the determinant of the coefficient matrix is different from zero, then we conclude that $\psi=0$ and $\psi^{a}=0$ and then the map $\mathcal{F}_{K}$ is injective. By direct inspection it can be checked that these coefficients are the same found in equations (41)-(43) in [2].

Second, consider the vector equation and the contraction of the two indices tensor equation with $\zeta^{a}$. This constitute a linear algebraic system of two vector equations for the variables mentioned in paragraph above, and $2 \zeta^{a} K_{(a} \psi_{b)}$ and $K_{b} \psi$. Because of injectivity, the only solution for all these variables is zero and so, the determinant of the coefficient matrix is different from zero. It can be checked, by direct inspection, that these coefficients are the same found in equations (45)-(46) in [2].

Finally, consider the two indices tensor equation. It constitutes a linear algebraic two indices equation for the variables mentioned in the previous paragraph and 
$K_{(a} \psi_{b)}$. Because of the injectivity, the only solution for all these variables is zero and so, the determinant of the coefficient matrix is different from zero. By direct inspection it can be checked that these coefficients are the same found in equations (48) in [2].

\section{APPENDIX B: GENERAL STABILITY THEOREM}

Consider the Cauchy problem for a first order system of partial differential equations,

$$
\begin{aligned}
\frac{\partial v}{\partial t} & =\left(A_{0}^{j}+\varepsilon A_{1}^{j}(v, \varepsilon)\right) \frac{\partial v}{\partial x^{j}}+\left(B_{0}+\varepsilon B_{1}(v, \varepsilon)\right) v \\
v(t & =0)=f\left(x^{j}\right)
\end{aligned}
$$

where $v: \mathbf{R} \times \mathbf{T}^{s} \rightarrow \mathbf{R}^{n}, A_{1}^{j}(v, \varepsilon)$ and $B_{1}(v, \varepsilon)$ are smooth $\left(C^{\infty}\right)$ functions of their arguments, and $f(x): \mathbf{T}^{s} \rightarrow \mathbf{R}^{n}$ is also smooth. Let $P$ denote the projector in the kernel of $B_{0}$. For the solution $v$ of $(\overline{\mathrm{B} 1})$ we define $v^{(0)}=P \hat{v}(0, t)$ and $w=v-v^{(0)}$. The stability theorem proved in [0] states [12].

Theorem 2 Suppose that the matrices $A_{0}^{j}, B_{0}$, and $A_{1}^{j}$ are hermitian, and the system (B1) satisfy the "relaxed stability eigenvalue condition", ie., the following conditions hold.

(i) There is a constant $\delta>0$ such that the eigenvalues $\lambda(k)$ of the symbol $i A_{0}^{j} k_{j}+$ $B_{0}$ satisfy $\operatorname{Re}\{\lambda\} \leq-\delta$ for all $k \in \Omega, k \neq 0$.

(ii) The eigenvalues of $B_{0}$ satisfy either $\operatorname{Re}\{\lambda(0)\} \leq-\delta$ or $\lambda(0)=0$.

(iii) ker $B_{0} \subset \operatorname{ker} B_{1}$.

Then, for $0 \leq \varepsilon \leq \varepsilon_{0}$ with $\varepsilon_{0}$ small enough, the system (B1) is a contraction for $w$ in a suitable norm, equivalent to a Sobolev norm $H^{p}(p>s+2)$, and $v^{(0)} \rightarrow$ const. when $t \rightarrow \infty$.

The statement in the above theorem that the system is contraction means that there exists an $H$-norm, equivalent to the norm $H^{p}$, such that

$$
\frac{d}{d t}\|w\|_{H}^{2} \leq-(\delta+\mathcal{O}(\varepsilon))\|w\|_{H}^{2}
$$

this implies that, for $\varepsilon$ small enough, there exists a global (in time) smooth solution of the Cauchy problem (B1) such that there is an exponentially decaying bound for the Sobolev $H^{p}$ norm of the $w$-part of its solution, and the $v^{(0)}$ part goes to a constant when the time goes to infinity.

The only difference between the fluid equations (1) and (B1) is the presence of $N$ in front of the time derivative. This causes no difficulties, and the theorem above is applicable by a simple redefinition of the scalar product used. With this new scalar product, the eigenvalues problem for the fluid equations becomes

$$
\operatorname{det}\left(i N_{0}^{-1} N_{0}^{j} k_{j}+N_{0}^{-1} I_{0}-\lambda E\right)=0 .
$$


where $E$ is the identity matrix. Then, the conditions (i), (ii), and (iii) of the theorem 2 can be verified as is done in the proof of theorem 11.

\section{References}

[1] I. S. Liu, I. Müller, T. Ruggeri, Ann. Phys. 169, 191 (1986).

[2] R. Geroch and L. Lindblom, Phys. Rev. D 41, 1855 (1990).

[3] R. Geroch and L. Lindblom, Ann. Phys. 207, 394 (1991).

[4] R. Geroch, J. Math. Phys. 36, 4226 (1995).

[5] L. Lindblom, Ann. Phys. 247, 1 (1996).

[6] H. O. Kreiss and J. Lorenz, Initial-Boundary Value Problems and the NavierStokes Equations (Academic Press, 1989).

[7] H. O. Kreiss, O. E. Ortiz and O. A. Reula, submitted (1996).

[8] It is known that strict equilibrium states are more general, but they reduce to constant states when the space-time is $\mathbf{R} \times \mathbf{T}^{3}$ and flat.

[9] T. Kato, Perturbation Theory for Linear Operators (Springer Verlag, 1966). See also, G. W. Stewart and Ji-guang Sun, Matrix Perturbation Theory, Computer Science and Scientific Computing (Academic Press, 1990).

[10] A. Matsumura, Publ. Res. Inst. Math. Science, Kyoto Univ., 13, 349 (1977).

[11] H. O. Kreiss, O. E. Ortiz and O. A. Reula, Proceedings of the Pacific Conference on Gravitation and Cosmology, (World Scientific, 1996).

[12] A more general theorem without the condition of symmetry is also proved in [6]. For the present application it is enough to consider the symmetric case. 